\title{
MJN THE FAMILY CAREGIVERS' NEEDS ON CARE AT HOME WITH SEVERE TRAUMATIC BRAIN INJURY SURVIVORS: A REVIEW
}

\author{
Salizar binti Mohamed Ludin, Hamidah binti Othman* \\ Critical Care Nursing Department, Kulliyyah of Nursing, International Islamic University Malaysia (IIUM), \\ Malaysia
}

*Corresponding Author's Email: hamidahothman78@gmail.com

\begin{abstract}
This paper aims to review the literature with regards to the family caregivers of severe traumatic brain injury survivors and their needs for care at home. The search started by finding the relevant keywords from EBSCOhost: MeSH database. There were many related studies found by searching the following multiple databases. They are from Evidence Based Medicine (EBM) reviews@ Ovid, Journal @ Ovid Full text, Medline with Full text via EBSCOhost, CINAHL Plus with Full Text, Science Direct, and Google scholars' articles. The search terms were limited to publications ranging from 2010 to 2016. Several points were highlighted regarding the needs of family caregivers who had been taking care of traumatic brain injury survivors. It also provided information on the needs of family caregivers in terms of care, traumatic brain injury, TBI survivor complications, family caregiver's burden, caregiver challenges and the impact of traumatic brain injury to family caregiver's quality of life. Overall, the study shows that family caregivers face significant challenges toward care of TBI survivors. The review highlighted the support needed from the family caregivers, and other important facts about severe traumatic brain injury in order to understand the condition. Further research should focus on rehabilitation efforts on traumatic brain injury survivors.
\end{abstract}

Keywords: Traumatic Brain Injury, Family Caregivers, Needs, Rehabilitation, Home Based Care

\section{INTRODUCTION}

The literature on brain injury is focused primarily on victims of injury, reporting outcomes in terms of medical status, cognitive functioning, employment levels, and behavioral sequelae (Rabinowitz \& Levin, 2014). However, in recent years, rehabilitation specialists have become increasingly concerned about the impact of traumatic brain injury (TBI) on family systems (Giacino, Hodgesmith \& Graham, 2016). Severe TBI is a devastating injury for patients and their families as it causes an injury that will forever change their lives (Stocchetti \& Zanier, 2016). Rosenfeld et al., (2012) has stated that a proportion of severe TBI survivors-after prolonged hospital care-require long rehabilitation as they may have long-term physical, cognitive, and psychological disorders. Such disorders may disrupt previous relationships and preclude return to work, with severe economic and social impacts. Roozenbeek, Mass \& Menon (2012) stated that the global burden is such that TBI survivors have a lower life expectancy than the general population with significant variability in the physical, cognitive and social aspects of recovery, making the prognosis for the individual uncertain. Due to this uncertainty, families often exhibit intensive needs for information, emotional support and involvement in care, as they struggle to adapt to the changes in their life (Liu et al., 2015). The effects of brain injury often change roles and responsibilities within the family. Individuals with TBI often rely on caregivers for longterm and life-long assistance (Dillahunt-aspillaga et al., 2013). Caregivers and others close to a person with brain injury may struggle to cope with behavioral changes caused by the brain injury. This article introduces the relevant articles or journals related to family caregivers needs on care of traumatic brain injury survivors.

\section{METHODOLOGY}

\section{Literature Search Strategies}

The aims of this literature review are to find related articles based on the past studies on the needs of 
caregivers among severe traumatic brain injury survivors. This review also includes evidence report on associated factors of psychological, physical and emotional needs. Relevant research is evaluated and analysed to highlight the knowledge gaps as well as justify the relevance of this study. To avoid data overload, the literature review process is guided by specific questions and objectives.

\section{Objective of Literature Review}

\section{The objectives of the literature review are as follows:}

1. To explore the needs of the caregivers among severe traumatic brain injury survivors.

2. To explore the challenges of the caregivers among severe traumatic brain injury survivors.

3. To explore experience of the caregivers among severe traumatic brain injury survivors.

4. To explore quality of life of the caregivers among severe traumatic brain injury survivors.

\section{Search Strategies}

The search started by finding the relevant keywords from EBSCOhost: MeSH database. The keywords used to retrieve the relevant studies were: TBI Survivors, family members' TBI, caregiver needs on care, care of critically ill patients, caregiver burden and quality of life. The results were further narrowed using keywords of 'caregivers' needs' and 'severe traumatic brain injury' or 'head injury'. There were many related studies found by searching the following multiple databases. They are from Evidence Based Medicine (EBM) reviews @ Ovid, Journal@ Ovid Full text, Medline with Full text via EBSCOhost, CINAHL Plus with Full Text, Science Direct, and Google scholar articles. The search terms were limited to publications ranging from 2010 to 2016.

The search was limited to studies published in the English language and full-text versions. The medical terms used were caregivers, severe traumatic brain injury, needs and care ("and," and "or") were used to narrow and widen the search. To increase the number of the articles using EBSCOhost and CINAHL Plus search engine, the author used the "related article" functions.

\begin{tabular}{|l|l|}
\hline Keyword & Similar words \\
\hline Caregiver needs & $\begin{array}{l}\text { Caregiver required, family } \\
\text { needs, family caregiving }\end{array}$ \\
\hline $\begin{array}{l}\text { Traumatic brain } \\
\text { injury }\end{array}$ & $\begin{array}{l}\text { Head injury, severe traumatic } \\
\text { brain injury, brain injury }\end{array}$ \\
\hline Quality of Life & Health lifestyle, health status \\
\hline Home Based Care & Home care, Rehabilitation care \\
\hline
\end{tabular}

To maintain the focus of the review was maintained by limiting the search results and inclusion and exclusion criteria were formulated. The research data had to be predominantly on (a) family caregivers of traumatic brain injury survivors (b) home base, community or hospital setting (c) method of the study such as a mix and quantitative method particularly qualitative, qualitative and cross-sectional study. While the exclusion criteria were (a) report on patients' outcome or prevalence study (b) studies on children with TBI.

\section{Search Findings}

The databases yielded 2058 potential articles of studies relevant to the research topic. After removal of duplicate articles and screening relevant articles, 1921 articles were excluded. Based on abstract, 137 potentially relevant articles were identified and screened by the author. The author then excluded 130 of the articles. Out of 129 articles, of 33 articles only abstract was available, 13 articles were with overlap studies, which means some articles are the same but has been published in many databases, 52 duplicate articles, 22 articles focus on patients and not on the family needs, 3 evidence-based practice and 6 inadequate information about outcome measures. Eventually, 7 articles were selected; there are 3 cross sectionals, 3 qualitative and 1 mixed method. The data extraction from the articles was done independently by both reviewers.

The review for mixed method study was based on Pluye et al., (2011) while the review for quantitative and qualitative studies are based on guidelines for critiquing research by Coughlan, Cronin \& Ryan (2013). The guidelines stated which elements were to be considered when appraising the strength and also mentioning limitation of the study.

\section{Data Extraction}

Based on the systematic review search in the three databases, there are only seven related articles included for appraisal. Among these articles, there are three articles on cross-sectional studies by Mazlan et al., (2016), Chayaput et al., (2014) and Dillahunt-aspillaga et al., (2013); three articles on qualitative studies by Keenan \& Joseph (2010), Carlozzi et al., (2015) and Webster, Taylor \& Balchin. (2015); and only one article on mixed method study by Mbakile-Mahlanza et al. (2016) to explore the needs of caregiver on functional and social outcomes. Additionally, the study on caregivers' needs have been conducted worldwide. Most of the studies were conducted in the United States of America including Florida and Michigan. Besides that, there are 3 studies conducted in Ontario, Canada, South 
Africa and Melbourne, Australia. Meanwhile, only two studies were revealed to be done in Asian countries including one from Thailand and one from Malaysia. The study that was conducted in Malaysia was by Mazlan et al., (2016) on life satisfaction and strain among informal caregivers with TBI was a crosssectional study. However, there were no studies in Malaysia to explore the needs of caregivers of severe traumatic brain injury survivors' in this country.

\section{RESULTS}

\section{Literature Review}

A cross-sectional study was done by Dillahuntaspillaga et al. (2013) to evaluate the strain among the caregivers due to unmet support needs. The study duration was not mentioned in this study. The 53 caregivers of traumatic brain injury participated in this study. This study found significant differences about the unmet needs of caregivers. As a conclusion, the availability of needs resources such as 'patients' skill training', 'a wider network of support', 'support group of fellow caregivers', 'trustworthy respite care', 'caregiver advocate', 'a guide /glossary for TBI related medical terminology' need to be emphasized. But, the sample size in this study is small and cannot be generalized to other populations. A cross-sectional study by Chayaput et al., (2014) focused on the coping and health problems of caregivers. The time frame was not mentioned in this study, but the researcher stated that data collection was done after the patients were discharged from the hospital. A total of 85 caregivers were involved in this study and the majority were female. The researcher mentioned that the culture in Thailand denotes that majority female does all the housework including looking after the patients who were discharged home. The instruments used for gathering the data was a Thai version of the coping and adaptation processing scale-short form. No statistical differences were found between coping and health problems among the caregivers in this study. As a conclusion, further studies need to be done on coping among caregivers of traumatic brain injury survivors.

The cross sectional study by Mazlan et al. (2016) was conducted to evaluate the life satisfaction and strain among informal caregivers of TBI patients. The representative of the study setting done by Mazlan et al. (2016) at the rehabilitation unit, University Malaya Medical Center, Kuala Lumpur Malaysia. A total of 141 participants were included in this study and the instrument used to evaluate the objectives were Life satisfaction questionnaire-9 and Caregiver strain index questionnaires. The conclusion in this study, from demographic data, is that the caregivers with lower income and caring for TBI patients with cognitive and neurobehavioral disturbances are at higher risk of developing strain. The cross-sectional study has limitations in finding the caregivers needs in-depth.

Therefore, a qualitative study was also done by Keenan (2010), Carlozzi et al. (2015) and Webster et al. (2015) to explore the caregiver needs among TBI survivors. The study by Keenan (2010) was conducted to identify the needs and determine if these needs change over time. The researcher mentioned in the study that the time frame for data collection was over 29 months and the study setting was at level 1 of the trauma academic health institution. The number of participants involved in this study was 25 caregivers and majority of the participants were female. The theme of the interview data was about the 'involvement on care', 'looking on the progress', 'meaning of life', 'the information needs', 'professional \& community support', 'uncertainty', 'Transition' and 'managing interpersonal issues'. As a conclusion, in this study, the large number of female caregivers may not reflect the reality of the male caregivers.

Another study conducted by Carlozzi et al., (2015) about health-related quality of life in caregivers of TBI survivors. The purpose of the study is also an integrated conceptual framework based on the results of the study. The conceptual framework was suitable with the study conducted. The study setting was at hospitals and the rehabilitation centers. There were 55 caregivers involved in this study with moderate to severe TBI. The researcher did not mention the outcome measures but the result in this study indicated that caregivers are more concerned about their social, emotional, physical and cognitive health. The domains of health related quality of life instrument is still not addressed in this study. Webster et al. (2015) did exploratory studies where the main objective is to examine the challenges among the caregivers with TBI. The data collection started in 2008 till 2013 with 175 participants; all the participants were then included in an interview session. The result showed the thematic analysis and the theme identification had 'lack of accessible information', content of required information, 'type of information and support', "need on development of S-plan' and 'counselling in support group'. The conclusion from this study is that the experiences of the caregivers help the researcher to develop the S-Plan. In Malaysia, there are no studies to explore the caregiver needs and to develop the module or package for caregivers of severe TBI survivors.

Over all the studies that were reviewed, one article on mixed method study was selected to be reviewed. The 
study was done by Mbakile-Mahlanza et al. (2016) to explore the needs of caregivers on functional and social outcomes. The study was conducted at the hospital and mostly at the participant's home. The study included the TBI survivors and their caregivers. Only 18 caregivers were willing to join the interview session. The data was continuously collected until data saturation was reached. The researcher chose the mixed method approach because it allowed the triangulation and substantiation of study finding. There was no mention of the instrument used for the qualitative part, but the results of the study were included. The quantitative results were displayed in a table. As for the qualitative results, the researcher highlighted the themes such as 'lack of information', 'heavy demands of caregivers', 'emotional distress', 'social isolation and lack of community support', 'financial burden', 'family devotion' and 'faith in god'. In summary, the caregivers face significant challenges toward care of TBI survivors and the rehabilitation efforts may use these results for further studies.

\section{Study Findings}

The findings were included in the study. There are several points that can be highlighted on the needs of caregivers who have been taking care of the traumatic brain injury survivors. The following section were reported on the caregivers needs on information, caregiver challenges, caregiver experience on burden and the impact of traumatic brain injury to caregivers' quality of life.

\section{DISCUSSION}

\section{Family Caregiver Needs}

Family Caregivers who have their needs assessed often feel acknowledged, valued, and better understood by practitioners. In case of providing quality care, recipient often requires an understanding of the family caregiver's situation and needs. However, these needs are frequently unidentified or unaddressed in the recipient's care plan. Rehabilitation access and outcome after severe traumatic brain injury identified TBI caregiver needs in an American population and the study found that the most important needs included information on the exact problems of the patient, access to someone who can answer questions about patient care in a truthful manner, access to professionals' advice to ensure the best care for the patient and respect from all health professionals. When caregivers are supported, they are better able to continue in this role, thereby reducing the cost of both public and private services. Effectively supporting caregivers can reduce burdens and health risks that can impede a caregiver's ability to provide care.

\section{Family Caregiver Challenges}

Caregivers play an essential role in the rehabilitation process of individuals with moderate to severe traumatic brain injury (TBI). Families are big, small, extended, nuclear, and multi-generational, with one parent, two parents, and grandparents. They live under one roof or many. A family can be as temporary as a few weeks or as permanent as forever. Families are formed by birth, adoption, marriage, or formed out of desire for mutual support. Family members nurture, protect, and influence each other. Families are dynamic and with a definite culture unto themselves, with different values and unique ways of realizing dreams. Together, families become the source of rich cultural heritage and spiritual diversity. Each family has strengths and qualities that flow from individual members and from the family as a unit. Families create neighbourhoods, communities, states, and nations. Sustaining a Traumatic Brain Injury result in family strain due to the significant impact of the injury that influence upon the role and function of individuals and their families at home and in the community (Dillahunt-aspillaga et al., 2013). The well-being of caregiver and families affects their ability and willingness to care for the injured person (Vangel, Rapport \& Hanks, 2011). Therefore, in addition to providing patient care, caregivers must also play a role in family support, through providing information and allowing to be near the patients (Soury-Lavergne et al., 2012). Health providers should recognize that parents and family members can offer important information related to their relative's diagnosis, treatment and safety (Moretz, 2010).

\section{Family Caregiver experience on burden}

The prevalence of TBI, the extensive and long-term process of rehabilitation, the expenses to the society and families associated with loss of productivity, loss of income, and medical costs; and the toll on emotional well-being necessitated an in-depth understanding of the coping and adaptive strategies utilized by TBI survivors and primary caregivers (Adams \& Dahdah, 2016).

\section{The impact of TBI to family caregiver of survivors' Quality of Life}

The concept of health-related quality of life (HRQOL) and its determinants have evolved since the 1980 s to encompass those aspects of overall quality of life that can be clearly shown to affect health - either physical or mental (Alonso et al., 2004). The interest in the outcome of 
patients with TBI is long-standing among practitioners and researchers. They have primarily focused on return to work and employment (Soury-Lavergne et al., 2012), especially with regards to severe states of disability, but has lately also included quality of life as a relevant end-point (Pluye et al., 2011). This change is due to the fact that recent epidemiological data show that many severe cases suffer from disabilities and dependencies after recovery. TBI survivors have a great impact not only on patients' lives but also on the lives of their family members/caregivers. Health-related quality of life is a subjectively evaluated construct that refers to the impact of the disease or disability or its treatment on one's physical, mental and social well-being. Behavioral and personality changes following TBI may devastate a caregiving family's function. The families' struggle to manage the behavior of TBI-patients which might become a primary determinant of family burden. Inadequate preparation of caregivers and patients to deal with the subsequent personality and behavioral changes is a major complaint. Family members may lack the skills to manage, cope, and provide care in TBI rehabilitation (Arango-Lasprilla et al., 2010).

\section{Flowchart of the Review Search}

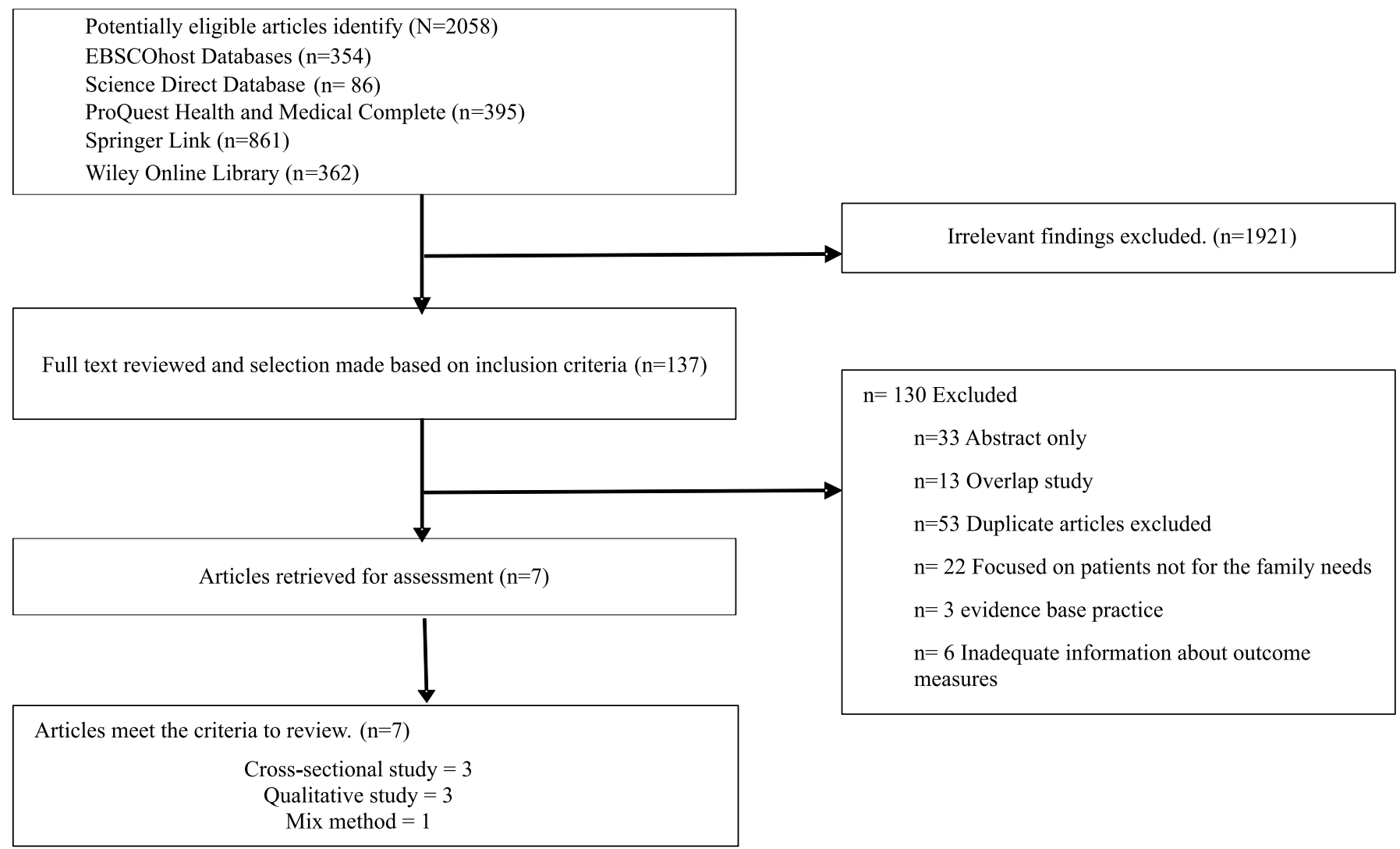

Figure 1: Flowchart of the Review Search

\section{Theoretical Framework Underpinning the Study}

There are several theories related with caregiver needs that drive the researcher to develop the concept of framework of the study. The important theories and models for the study will help the researcher to identify the suitable theory or model related with the study plan. A study done by Dillahunt-aspillaga et al., (2013) used the Stress process model of caregiving (SPMC). The SPMC focuses on the strong association between stress and the high demands of caregiving. This model also considers impacting factors such as psychosocial factors (coping and support) and demographic factors (e.g.: age and gender) to assess the caregiver's level of stress as well as potential resiliency factors. Additionally, caregiver quality of life (QOL) and stress appraisal are affected by family needs and social support and the SPMC highlights these factors (Figure 2). This study focuses on the stress among caregivers and the Stress Process Model of Caregiving for positive and negative caregiving experiences-whereby in this study-the researcher needs to focus on all aspects of need for caregivers, not only the stress component. 


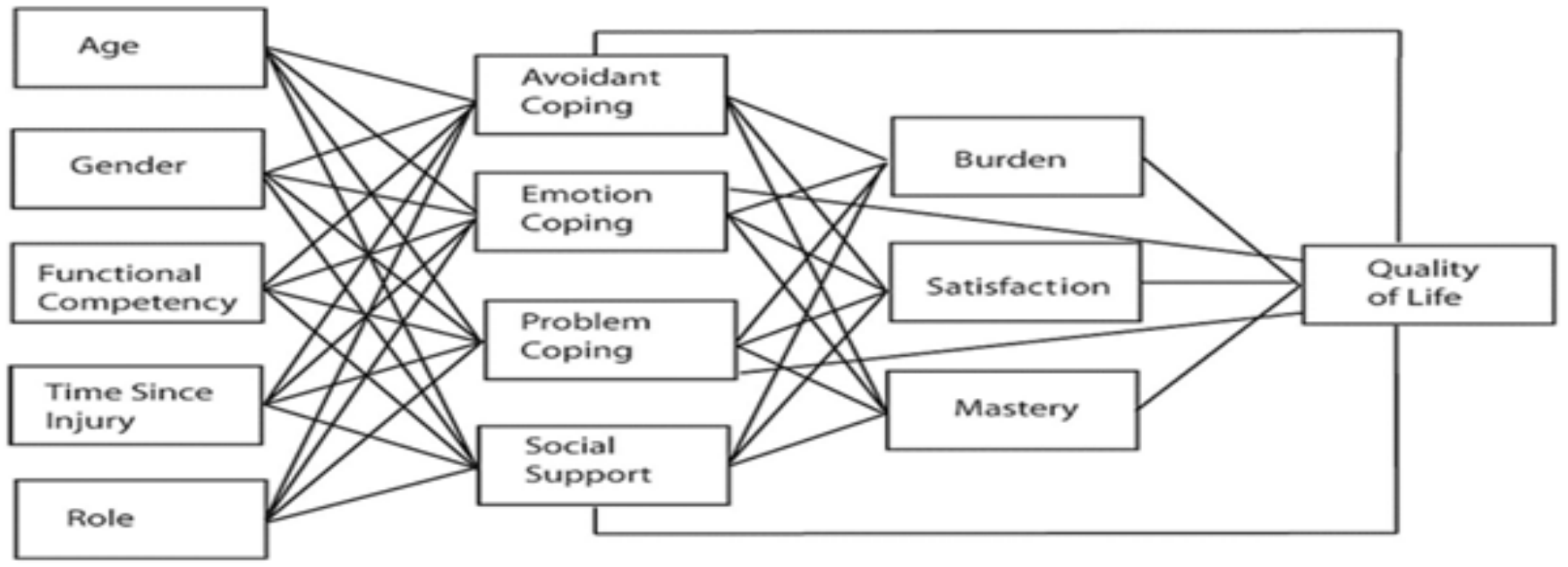

Figure 2: illustrates the stress process model of caregiving (SPMC) for individuals with TBI by Dillahunt-aspillaga et al. (2013)

In another study done by Chayaput et al., (2014), the researcher used the Roy adaptation model. According to the Roy Adaptation Model (RAM), individuals as adaptive bio-psychosocial systems will deal with challenging or threatening situations in their daily lives in order to survive. It will change the environments or stressors that a person perceives as difficult situations act as stimuli. Focal stimulus refers to a stressor with direct influence on behavioural outcomes. From the contextual stimuli, stressors affect the focal stimulus, but also play a role in promoting behavioural responses (Figure 3).

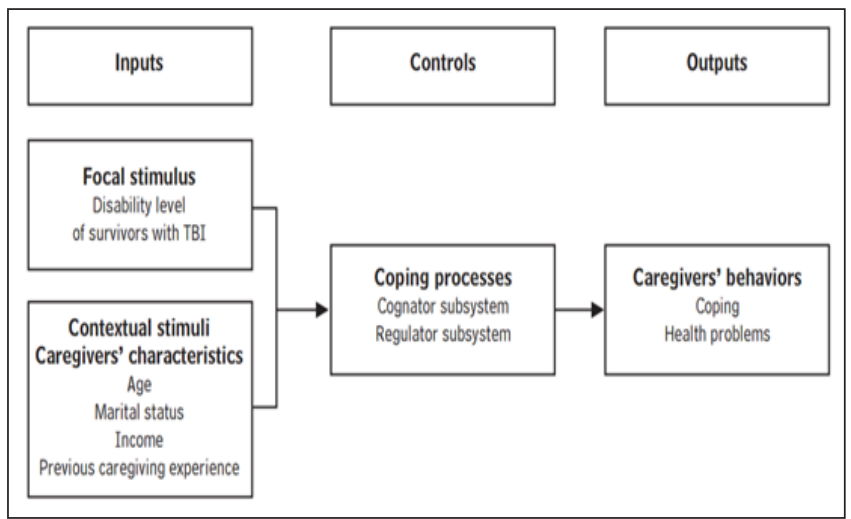

Figure 3: Diagram of correlations between variables of interest for caregivers relating to the RAM in this study

This study focused on 3 domains: Personal Data of Caregivers and Survivors with TBI, Coping and Health Problems of Caregivers and Disability Level of Survivors with TBI.

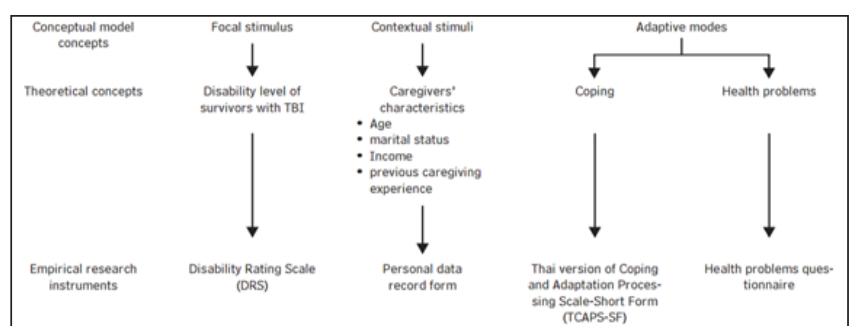

Figure 4: Conceptual-theoretical-empirical structure for the study on coping and health problems of caregivers of survivors with $T B I$

Health-related quality of life (HRQoL) is a multidimensional concept that includes domains related to physical, mental, emotional, and social functioning. It goes beyond direct measures of population health, life expectancy, causes of death and focuses on the health status impact on quality of life. Carlozzi et al. (2015) looked into the Health-related quality of life in caregivers of individuals with traumatic brain injury. The study had identified the aspects of health-related quality of life (HRQOL) that are relevant to caregivers of individuals with traumatic brain injury (TBI).

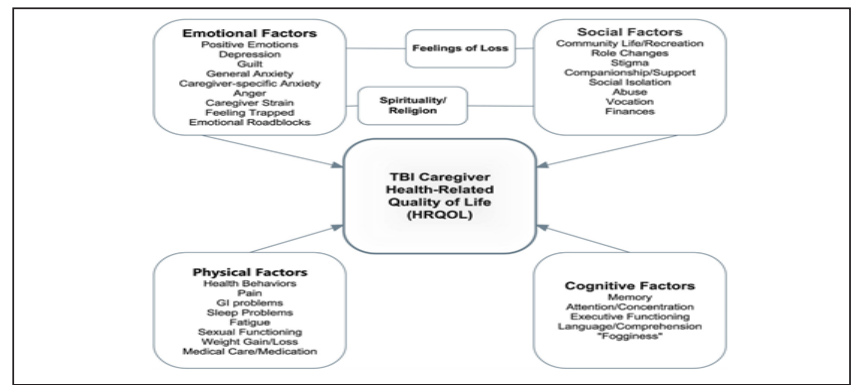

Figure 5: Conceptual model of factors related to the HRQOL of caregivers of persons with TBI 
After reviewing the literatures, the researcher has reached the conclusion that the above said conceptual models are used in this study to guide the researcher to identify the needs of caregivers and the conceptual framework helps the researcher to explore the needs of family caregivers on care of TBI survivors.

\section{CONCLUSION}

The review concluded that meeting family caregivers' needs can be achieved by exploring the specific needs and involving family caregivers to talk about their experience on care of severe traumatic brain injury survivors. Most studies also gave similar results in terms of the needs, burden and challenges. Apart from that, the quality of life is also affected among family caregivers during rehabilitation phase of TBIs. This study was able to hopefully help the family caregivers manage this difficult situation by recognizing the uniqueness of the family characteristics by providing care and support that allows them to adapt to the situation to look after their relative.

\section{REFERENCES}

Adams, D. \& Dahdah, M. (2016). Coping and adaptive strategies of traumatic brain injury survivors and primary caregivers. Neuro Rehabilitation, 39(2), pp 223-237.

Alonso, J., Ferrer, M., Gandek, B., Ware, J.E., Jr, Aaronson, N.K., Mosconi, P., Rasmussen, N.K., Bullinger, M., Fukuhara, S., Kaasa, S., Leplège, A.; IQOLA Project Group. (2004). Health-related quality of life associated with chronic conditions in eight countries: results from the International Quality of Life Assessment (IQOLA) project. Quality of Life Research, 13(2), pp 283-98.

Arango-Lasprilla, J.C., Quijano, M.C., Aponte, M., Cuervo, M.T., Nicholls, E., Rogers, H. L. \& Kreutzer, J. (2010). Family needs in caregivers of individuals with traumatic brain injury from Colombia, South America. Brain Injury, 24(7-8), pp 1017-1026.

Carlozzi, N. E., Kratz, A. L., Sander, A. M., Chiaravalloti, N. D., Brickell, T. A., Lange, R.T., Hahn, E.A., Austin, A., Miner, J.A. \& Tulsky, D.S. (2015). Health-related quality of life in caregivers of individuals with traumatic brain injury: Development of a conceptual model. Archives of Physical Medicine and Rehabilitation, 96(1), pp 105-113.

Chayaput, P., Utriyaprasit, K., Bootcheewan, S. \& Thosingha, O. (2014). Coping and Health Problems of Caregivers of Survivors with Traumatic Brain Injury. AQUICHAN, 14(2), pp 170-183.

Coughlan, M., Cronin, P. \& Ryan, F. (2013). Doing a Literature Review in Nursing, Health and Social Care (1 ${ }^{\text {st }}$ ed.). London: Sage Publications, Ltd.

Dillahunt-aspillaga, C., Jorgensen-smith, T., Ehlke, S., Sosinski, M., Monroe, D. \& Thor, J. (2013). Traumatic Brain Injury : Unmet Support Needs of Caregivers and Families in Florida. PLoS One, 8(12), e82896.

Giacino, J.T., Hodgesmith, M. \& Graham I.D. (2016). Rehabilitation Access and Outcome after Severe Traumatic Brain Injury: A TBI Model System-Sponsored Stakeholder Summit. United States Access Board, Washington, DC. Retrieved from: http://media-ns.mghcpd.org.s3.amazonaws.com/spauldingtbi/rehabilitation-access-andoutcome-after-severe-tbi-briefing-book.pdf

Keenan, A. \& Joseph, L. (2010). The needs of family members of severe traumatic brain injured patients during critical and acute care: a qualitative study. Canadian Journal of Neuroscience Nursing, 32(3), pp 25-35.

Liu, W., Zhu, J., Liu, J. \& Guo, Q. (2015). Psychological state and needs of family member caregivers for victims of traumatic brain injury: A cross-sectional descriptive study. International Journal of Nursing Sciences, 2(3), pp 231-236.

Mazlan, M., Ghani, S.Z., Tan, K.F. \& Subramanian, P. (2016). Life satisfaction and strain among informal caregivers of patients with traumatic brain injury in Malaysia. Disability and Rehabilitation, 38(22), pp 2198-2205. 
Mbakile-Mahlanza, L., Manderson, L., Downing, M. \& Ponsford, J. (2016). Family caregiving of individuals with traumatic brain injury in Botswana. Disability and Rehabilitation, 39(6), pp 559-567.

Moretz, J.G. (2010). Strengthening patient- and family-centered care: learning through webinars. Pediatric Nursing, 36(3), pp 168-170.

Pluye, P., Robert, E., Cargo, M., Bartlett, G., O’Cathain, A., Griffiths, F., Boardman, F., Gagnon, M.P. \& Rousseau, M.C. (2011). Proposal: A mixed methods appraisal tool for systematic mixed studies reviews. Montréal: McGill University,(Part I), pp 1-8.

Rabinowitz, A.R. \& Levin, H.S. (2014). Cognitive Sequelae of Traumatic Brain Injury. Psychiatric Clinics of North America 37(1), pp 1-11.

Roozenbeek, B., Mass, A.I.R. \& Menon D.K. (2012). Changing patterns in the epidemiology of traumatic brain injury. Nature Reviews Neurology, 9(4), pp 231-236.

Rosenfeld, J.V., Maas, A.I., Bragge, P., Morganti-Kossmann, M.C., Manley, G.T. \& Gruen, R.L. (2012). Early management of severe traumatic brain injury. The Lancet, 380(9847), pp 1088-1098.

Soury-Lavergne, A., Hauchard, I., Dray, S., Baillot, M. Lou, Bertholet, E., Clabault, K., Jeune, S., Ledroit, C., Lelias, I., Lombardo, V., Maetens, Y., Meziani, F., Reignier, J., Souweine, B., Tabah, A., Barrau, K. \& Roch, A; Société de Réanimation de Langue Française (SRLF) (2012). Survey of caregiver opinions on the practicalities of familycentred care in intensive care units. Journal of Clinical Nursing, 21(7-8), pp 1060-1067.

Stocchetti, N. \& Zanier, E.R. (2016). Chronic impact of traumatic brain injury on outcome and quality of life: a narrative review. Critical Care (London, England), 20(1): 148.

Vangel, S.J.J., Rapport, L.J. \& Hanks, R.A. (2011). Effects of family and caregiver psychosocial functioning on outcomes in persons with traumatic brain injury. The Journal of Head Trauma Rehabilitation, 26(1), pp 20-29.

Webster, J., Taylor, A. \& Balchin, R. (2015). Traumatic brain injury, the hidden pandemic: A focused response to family and patient experiences and needs. South African Medical Journal, 105(3), pp 195-198. 